\title{
Comments on 'Progressive atrophy in a deformed liver as a contributor to sigmoid volvulus'
}

\author{
Sabri Selcuk Atamanalp ${ }^{1}$
}

Received: 24 November 2020 / Accepted: 3 December 2020 / Published online: 17 January 2021

(c) Japanese Society of Gastroenterology 2021

\section{Dear Editor,}

I read with interest the report titled 'Progressive atrophy in a deformed liver as a contributor to sigmoid volvulus' written by Zenda et al. [1], who presented a case with sigmoid volvulus (SV) and medial segment liver atrophy (LA). SV, a rare colonic obstruction worldwide, is relatively common in Eastern Anatolia, my practicing area [2]. We have a 1,030case experience with SV over a 54-year period from June 1966 to July 2020, which is the largest single-center SV series over the world [3]. In light of this experience, I want to discuss herein the possible mechanisms of LA in SV.

Although there are limited individual reports on the association of SV and LA [1, 4], unfortunately, the cause and effect relation is not clearly identified in the literature [5]. Some authors suggest LA as a cause of SV and accept a relatively enlarged intraabdominal volume as a predisposing factor, which easily allows the rotation of the sigmoid colon resulting in SV [4]. However, there is a relatively adverse and complex mechanism in the development of SV [6]. Sigmoid colon occasionally twists physiologically and untwists spontaneously. In SV, the main pathology is the prevention of the untwisting, which is known as 'entrapment'. The main cause of the entrapment is the enlargement of the twisted loop due to the gas generation. Additionally, a relatively narrowed intraabdominal volume as seen in men or pregnant women, in whom SV is relatively common, helps to the process. As a result, in my theoretical opinion and clinical experience, LA may be a result, which arises from the chronic enlargement of the sigmoid colon. Although the authors think acute abdominal distress arising from SV in addition to the impairment of flow in the portal vein and/ or biliary system as the main causes of LA, I wonder about

Sabri Selcuk Atamanalp

ssa@atauni.edu.tr

1 Department of General Surgery, Faculty of Medicine, Ataturk University, 25040 Erzurum, Turkey their theoretical opinion as well as clinical experience on my theory.

I congratulate the authors and look forward to their reply on my comments.

\section{Compliance with ethical standards}

Conflict of interest Sabri Selcuk Atamanalp declares that he has no conflict of interest.

Human rights All procedures followed have been performed in accordance with the ethical standards laid down in the 1964 Declaration of Helsinki and its later amendments.

Informed consent Informed consent was obtained from all patients for being included in the study.

\section{References}

1. Zenda T, Araki I, Hamano N, et al. Progressive atrophy in a deformed liver as a contributor to sigmoid volvulus. J Clin Gastroenterol. 2019;13:632-3.

2. Atamanalp SS. Sigmoid volvulus: an update for Atamanalp classification. Pak J Med Sci. 2020;36:1137-9.

3. Atamanalp SS. Sigmoid volvulus: the first one thousand-case single center series in the world. Eur J Trauma Emerg Surg. 2019;45:175-6.

4. Barral M, Lassalle L, Dautry R, et al. Volvulus of the sigmoid colon is associated with hypotrophy of the left lateral segment of the liver and the absence of sigmoid diverticulum. Diagn Interv Imaging. 2018;99:247-53.

5. Web of Science. Sigmoid volvulus. http://apps.webofknowl edge. $. \mathrm{m} /$ Search.do? product $=$ WOS $\&$ SID $=E 1$ Xay4za7I5tRn 1 jfKs\&search_mode $=$ GeneralSearch\&prID=b87b340e-8d01-4ce790cb-52b9eef1f36a. Accessed 15 Oct 2020.

6. Atamanalp SS. Sigmoid volvulus. Eurasian Med J. 2010;42:144-9.

Publisher's Note Springer Nature remains neutral with regard to jurisdictional claims in published maps and institutional affiliations. 